\title{
Characterization of GEMINI, a 16-channels programmable readout interface for Triple-GEM detectors in 180nm CMOS
}

\author{
Luca Mangiagalli ${ }^{1}$ \\ University of Milano - Bicocca \\ Piazza della Scienza, 3, Milano \\ Italy \\ E-mail:l.mangiagalli@campus.unimib.it
}

\section{Gabriele Croci}

University of Milano - Bicocca, INFN

Marcello De Matteis

University of Milano - Bicocca

Diego Tagnani, Giovanni Corradi

Laboratori Nazionali di Frascati

Fabrizio Murtas

CERN, INFN

\section{Giuseppe Gorini}

University of Milano - Bicocca, INFN

\section{Andrea Baschirotto}

University of Milano - Bicocca

\begin{abstract}
The recent advances in GEM detector development has led to the requirement of a custom readout to fully exploit the advantages of this technology. GEM detectors can be realized with various shapes, also irregular, and high number of channels. GEMINI has been specifically designed to work with Triple-GEM detectors and it integrates 16 channels to perform readout with both analog and digital signal with Time over Threshold. GEMINI also allows to set a different threshold for every channel to compensate channel inhomogeneity. This work compares simulations with lab measurements and presents results of the imaging of an X-ray source performed with GEMINI.
\end{abstract}

Topical Workshop on Electronics for Particle Physics (TWEPP2018)

17-21 September 2018

Antwerp, Belgium

\section{${ }^{1}$ Speaker}




\section{Introduction}

Triple-GEM detectors are currently under development for application in imaging with neutrons and X-ray especially where radiation hardness requirements are too high for silicon detectors. Triple-GEM detectors employ the Gas Electron Multiplier (GEM) technology [1] and constitute a promising development. In particular many in many applications of the GEM detectors, like [2],[3],[4],[5], huge advantages can be obtained by the employment of TripleGEMs, as studied in [6],[7],[8]. Development of Triple-GEM also showed the need of a dedicated interface that could substitute integrated interfaces employed with GEM detectors in the past [9], [10] to meet the new requirements in terms of performance and usability. Indeed, some of the advantages of Triple-GEM detectors constitute challenges for the readout system. The readout from metal pads, in first place, exposes the electronics to the risk of destructive events deriving from discharge. Flexible shape and size inherited from GEMs means having channels with variable input capacitance, up to $40 \mathrm{pF}$, even in the same detector. Triple-GEM detectors detect incident particles thanks to gas ionization and amplifies the signal with three GEM layers collecting on metal pads the electron generated. Hence, readout circuitry is fed with current pulses approximately $10^{-7} \mathrm{~s}$ long. Considering the expected operating conditions the readout must sustain count rates up to $5 \mathrm{Mcps}$ in an input range between $30 \mathrm{fC}$ and $500 \mathrm{fC}$.

\section{GEMINI}

GEMINI [11] is an integrated interface designed specifically for Triple-GEM detectors in CMOS 180nm. It integrates 16 readout channels with a 9-bit threshold system that allows independent programming for each channel. A calibration system is also integrated to compensate process and temperature variations. Programming of GEMINI is possible thanks to a $\mathrm{I}^{2} \mathrm{C}$ interface. In Figure 1 a block diagram of a single channel of GEMINI is shown. A Charge Sensitive Preamplifier with sensitivity of approximately $1 \mathrm{mV} / \mathrm{fC}$ is implemented using a capacitive array that allows tuning of capacitance with the calibration system. The voltage signal generated is compared with the threshold set with the 9-bit DAC thanks to a Discriminator. The LVDS output driver allows fast transmission of digital data generated and an analog buffer allows to monitor the output of the Charge Sensitive Preamplifier during testing phase.

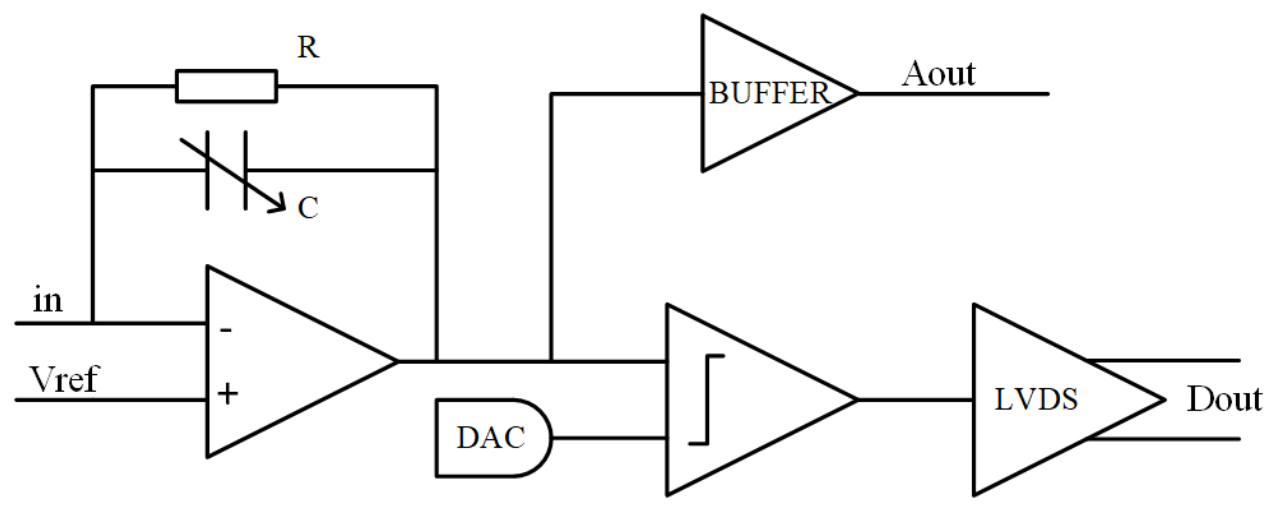

Figure 1 Block diagram of a channel from GEMINI. 
Considering the length of current pulses from the detector and the high counting rate required the design of the time constant of the Charge Sensitive Preamplifier has a fundamental role in the overall performance of the channel. This means that the response of the Charge Sensitive Preamplifier will be very fast, and it must be able to reproduce the shape of the signal generated by the detector.

\section{Measurements}

Characterization of GEMINI channel has been performed generating current pulses with a passive network stimulated with a voltage step. This method allowed fine tuning of input charge, adjusting voltage step amplitude, and the simulation of detector capacitance. In the next sections results from the analog characterization of the channel will be shown and data collected from Time over Threshold characterization will be presented.

\subsection{Channel characterization}

Linearity of the relationship between voltage signal amplitude and input charge is critical to preserve the information on integrated charge. For this reason, the characterization of GEMINI started from a verification of this relationship in the whole input range. A plot of analog output peak value, measured from signal baseline, is shown in Figure 2. Here, a comparison of data obtained from simulations and from measurements is done. A linear fit performed on measurement data shows the linearity of the response of GEMINI channel in the whole operating range with a sensitivity of approximately $0.9 \mathrm{mV} / \mathrm{fC}$.

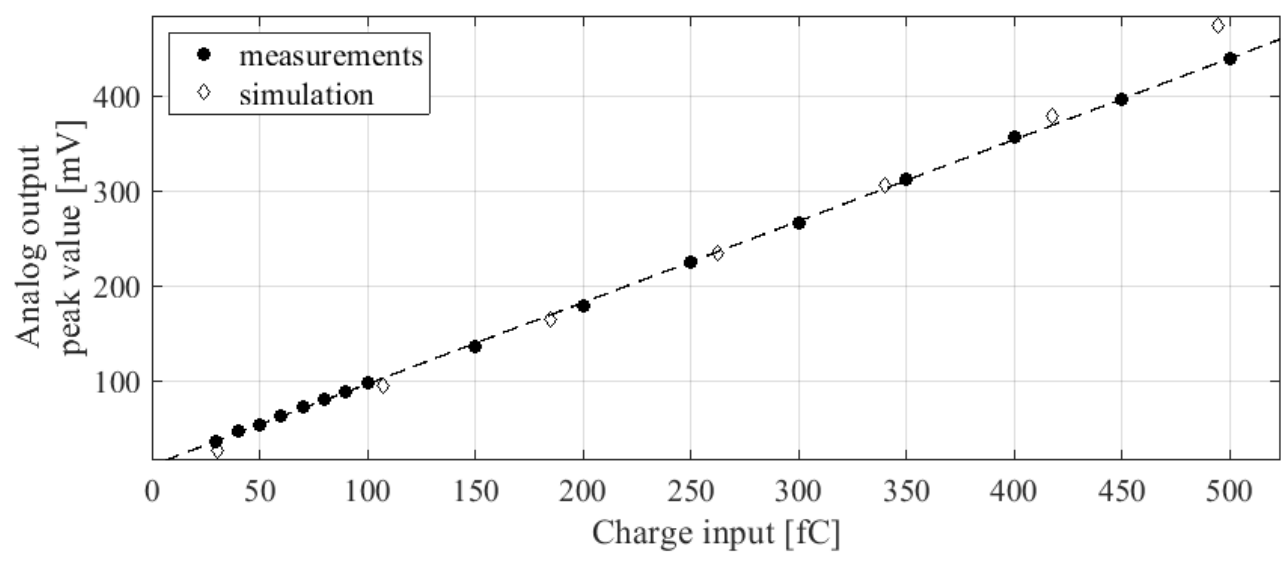

Figure 2 Plot of analog output peak value for various input charge in the expected operating range.

\subsection{Time over Threshold}

The Time over Threshold (ToT) feature has been tested acquiring single waveforms from the LVDS output and calculating the average signal length from 10000 samples for every data point. In Figure 3 a plot of ToT data is shown. The three series of points shown represent the characteristics obtained with different threshold values. As can be seen in the plot, higher thresholds give lower ToT values, decreasing the sensitivity of this measurement. Higher thresholds also give lower probability of detecting fake events from noise background. An accurate setting of threshold value is hence crucial to maximize the performance using this readout system. The behaviour visibile in Figure 3 is determined by the shaper of the peaks generated by 
the detector and for this reason the ToT characteristics varies in slope while varying the threshold.

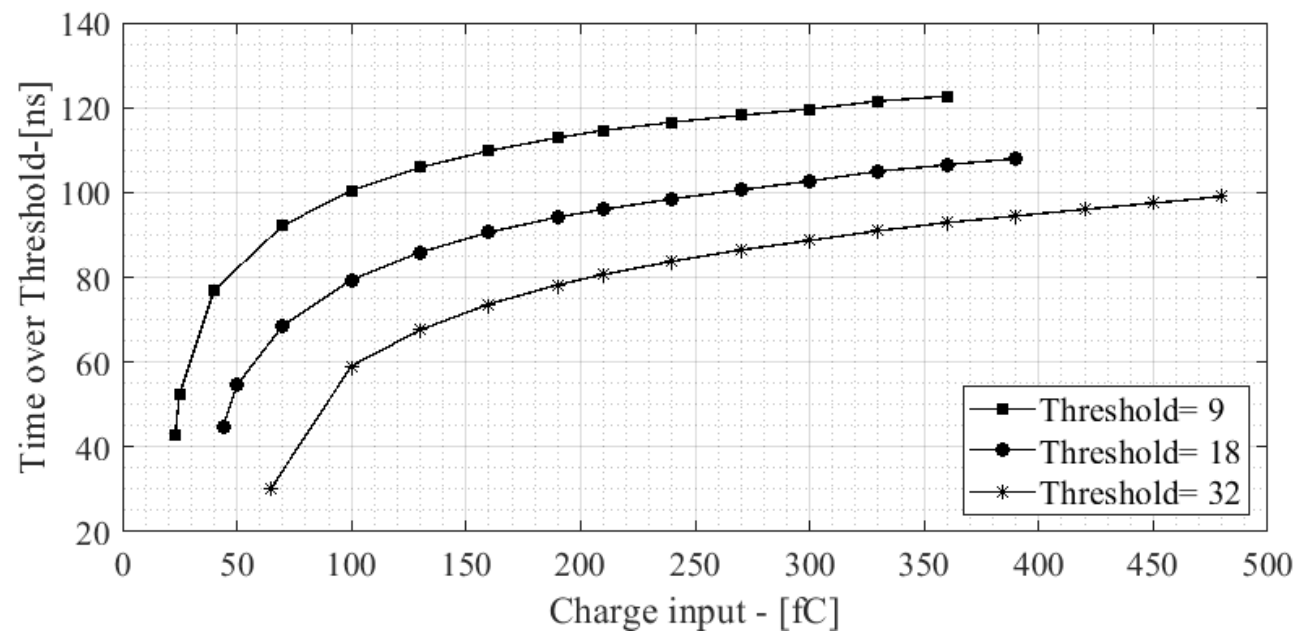

Figure 3 Plot of Time over Threshold measurement for various input charge values with three different threshold values.

\subsubsection{Imaging}

In Figure 4 an image acquired with GEMINI chips from a test detector is shown. To irradiate the detector a $\mathrm{Fe}^{55}$ source has been used. The shape of the circular test source is clearly visible, indicating the absence of patterns generated by the readout system. Even if the test detector used has a square shape, corner pads are bigger for area filling reasons. This results in higher counts on channels in the corners, as can be seen in Figure 4. However, GEMINI shows to be able to manage different pad size without compromising the overall image quality.

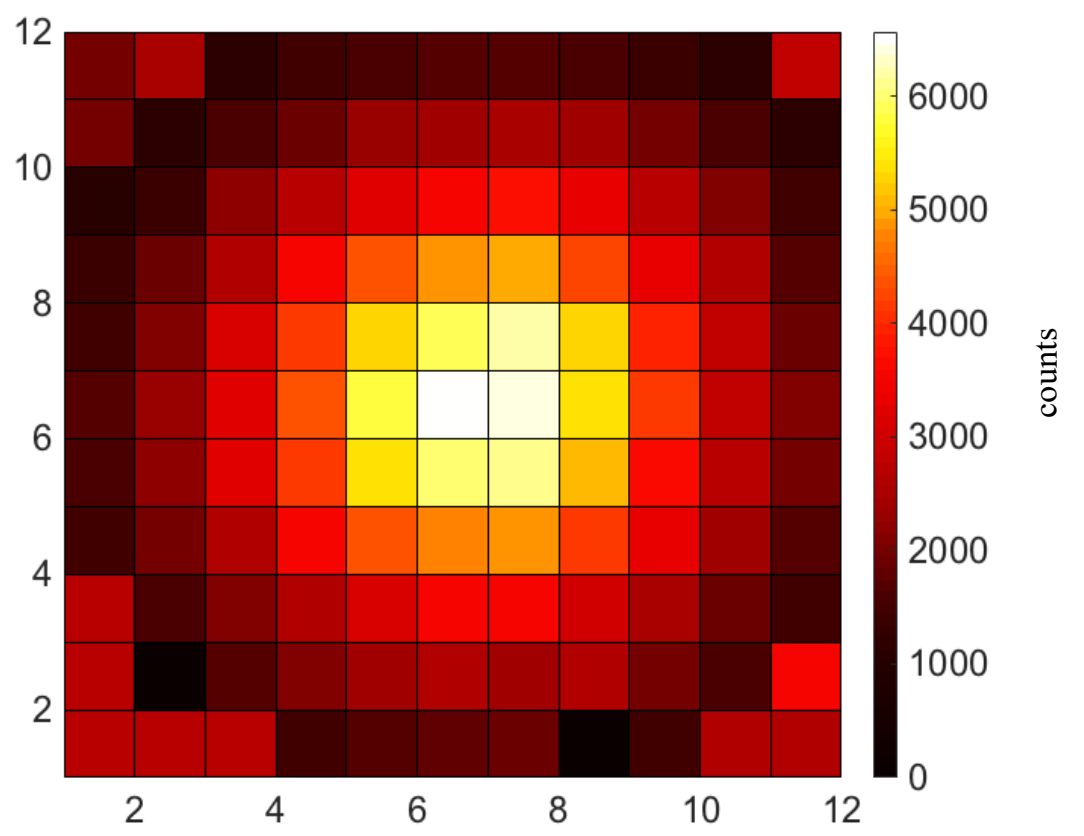

Figure 4 Image acquired with with GEMINI chips mounted on a test detector irradiated with a Fe $e^{55}$ source 


\section{Conclusions}

Results obtained during the characterization of GEMINI have been shown. Single channel performance in charge integration has been verified and a linear behavior in the whole input range has been observed. Time over Threshold functionality has also been tested and performance dependence from threshold set described. GEMINI has also been tested with a laboratory source to acquire some images useful to understand system overall performance.

\section{References}

[1] F. Sauli, GEM: A new concept for electron amplification in gas detectors, Nuclear Instruments and Methods in Physics Research Section A: Accelerators, Spectrometers, Detectors and Associated Equipment 386.2-3 (1997): 531-534.

[2] Inuzuka, M. "Development of a GEM detector for X-Ray radiography of cultural properties." Nuclear Science Symposium Conference Record, 2007. NSS'07. IEEE. Vol. 1. IEEE, 2007.

[3] Costa, Enrico, et al. "An efficient photoelectric X-ray polarimeter for the study of black holes and neutron stars." arXiv preprint astro-ph/0107486 (2001).

[4] Pacella, D., et al. "Ultrafast soft x-ray two-dimensional plasma imaging system based on gas electron multiplier detector with pixel readout." Review of Scientific Instruments 72.2 (2001): 13721378.

[5] Buzulutskov, A., et al. "The GEM photomultiplier operated with noble gas mixtures." Nuclear Instruments and Methods in Physics Research Section A: Accelerators, Spectrometers, Detectors and Associated Equipment 443.1 (2000): 164-180.

[6] Simon, Anna, et al. "A scintillating triple GEM beam monitor for radiation therapy." Nuclear Science Symposium Conference Record, 2005 IEEE. Vol. 5. IEEE, 2005.

[7] Murtas, Fabrizio, et al. "Applications in beam diagnostics with triple GEM detectors." Nuclear Instruments and Methods in Physics Research Section A: Accelerators, Spectrometers, Detectors and Associated Equipment 617.1 (2010): 237-241.

[8] Valente, Paolo, et al. "Triple-GEM detectors for electron, proton and neutron beam diagnostics." Nucl. Instrum. Meth. A (2010).

[9] Balla, A., et al. "GASTONE: A new ASIC for the cylindrical GEM inner tracker of KLOE experiment at DAFNE." Nuclear Instruments and Methods in Physics Research Section A: Accelerators, Spectrometers, Detectors and Associated Equipment604.1 (2009): 23-25.

[10] Moraes, D. CARIOCA-a fast binary front-end implemented in $0.25 \mathrm{~m}$ CMOS using a novel currentmode technique for the LHCb muon detector. No. LHCb-TALK-2000-050. 2000.

[11] Pezzotta, A., et al. GEMINI: A triple-GEM detector read-out mixed-signal ASIC in 180nm CMOS. In Circuits and Systems (ISCAS), 2015 IEEE International Symposium on (pp.1718-1721). 